\title{
An loT based Smart Power Mangement System for Technical University
}

\author{
Khalid A. Fakeeh, PhD \\ King Abdullaziz University \\ Jeddah, Saudi Arabia
}

\begin{abstract}
IoT has been a great use in the modern world; the importance and usage of IoT's are increasing day by day. There is lots of research going into IoT based power management systems. The increase in power requirement has pushed the researchers and industrialist to design low power systems. The wastage of energy is a very serious concern; this paper proposes a novel method to reduce the wastage of power in technical universities by the power distribution agencies across the world. The system consists of thermal sensing and IoT enabled microcontrollers for the working.
\end{abstract}

\section{Keywords}

IoT,Thermal sensing,IoT enabled microcontrollers

\section{INTRODUCTION}

Internet is an interconnection of computers all over the world. It follows TCP/IP protocols. Internet links billion of devices worldwide, and is used to send, receive data all over the world. It was first developed in the USA. The first World Wide Web (WWW) was developed at CERN, and because of this it became accessible to the whole world. Internet has vast uses and applications in many fields and domain. One of the important applications of the internet is IoT. IoT is interconnection of physical objects, vehicles etc. mixed with various other fields like embedded systems, sensors, software which helps to collect, transfer and exchange information. One of the main applications of IoT is monitoring information from a remote location.

Power management is an important aspect towards the development of the nation. Most of the countries are trying to develop and execute various projects to conserve energy. Power should be utilized efficiently, and for that we need systems which consume power efficiently. The bulbs being replaced by CFL are one of the examples of such a project being carried out by electricity distributors all over the world.

But the energy consumption is increasing rapidly, and the energy is not sufficient enough to meet the rising demand. Many of the countries have renewable energy source, but are not utilizing it. The usage of hydroelectric power which was the major source of energy in the twentieth century is now being replaced by nuclear energy. Since nuclear plants have many security issues. This increases the installation cost of the reactor.

When there is such an energy crisis, one possible solution is efficient utilization of energy. This can be done only if every individual contributes to power saving. That is they should not waste power unnecessarily .only if this is done there can be efficient utilization of energy and energy crisis can be reduced. So the society needs to be educated on power saving and power wastage reduction. A further step towards power saving is by imposing penalty for power wastage. It can be imposed on industries and households.
IoT based power management system is an effective method for power management. The recent development in internet of things has helped researchers and industrialist to identify its role in energy in management system. The usage of IP (Internet Protocol) enabled service has now proven that it can be used to save energy.

In older days internet had a limited applications. Now internet is a chain of network connection in which the numbers of connected devices are increasing rapidly. At present the internet is used to access, process and command real time parameters from remote locations. The implementations of IoT based systems are inexpensive. But it will not be cost effective and efficient if implemented improperly. In olden days, for home automation, large number of sensors is used to control electrical appliances. The large number of sensors used is not cost effective. Since each appliance requires unique sensor the cost and the power consumed increases as the number of devices increases. In modern IoT systems the large number of sensors can be replaced by few sensors and can be put in a single platform thus consuming both power and energy. The context aware systems are designed for such purposes which allow the IoT to work effectively. Different models of context aware system exist; they are object oriented and antilogy base [1]. Impress context management enables rapid development of the context aware IoT system which can be applied in many areas of interest. Recent trend use image processing to perform context management [2]. A camera acts as an input to the image processing system. The camera monitors human activities and the IoT systems perform the required adjustments based on the input. The cameras can be replaced by thermal sensors, which are cheaper than cameras [3]. The thermal sensors can detect infrared waves emitted by humans. Based on the IR signal they can detect the presence of humans and monitor their activities. Thermal sensors are more cost effective compared to cameras. They also do not need complex image processing. Instead simple filtering and transformations are sufficient. So thermal sensors are considered better option when considering cost as a main aspect. 


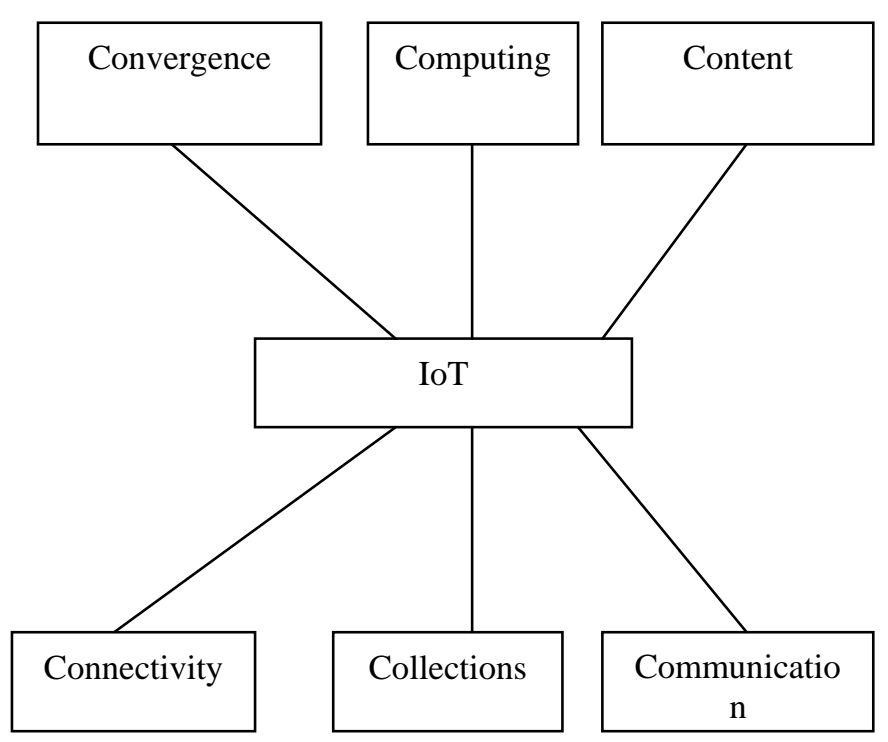

Figure 1: a high level view of IoT

The successfulness of an IoT system also depends on the environment where it is installed. For instance if it is installed in a forested area, the path loss during transmission will be greater than line of sight communication[5]. Another important aspect of IoT system is its adaptation for different season. In different season the sensor constraints such as temperature are different. The IoT based system should be able to handle and change according to such changes. Only then the IoT system can be put to real time application. Thus handling seasonal variation becomes one of the major aspects of an IoT based system. Similarly the physical parameters at different regions are different. When the IoT systems are used worldwide, the physical parameters are completely different. So along with seasonal constraints the IoT must also be able to handle regional constraints.

IBM has now introduced a framework for autonomic computing [6]. It is aimed at easier management of systems. The framework consists of two units. They are managed resource and autonomic manager. The managed resource is simple entity and consists of sensors and effectors. The sensor senses the environment and collects the data. The effectors are the interface which the device uses to manipulate the environment. The autonomic manager on the other hand is more complex and provides centralized control and performs data analysis. The centralized control includes monitoring of data and reporting. An environment will be monitored and the data will be collected. The data is filtered, managed and then reported. The analyzing part models the situation and learns about the environment. This helps in predicting the future situation. Further mechanisms are provided to guide the action based on the policies in order to achieve the required objective. These plans will be further executed. Thus it acts as a self-learning adaptive IoT based system. These systems would be much more efficient and reliable.

The IoT platforms are specific to their application. In a single IoT platform, various applications are provided. The application can include business, enterprise, company and Government related [4]. All together it can perform as a generic platform for entire city or country.

\begin{tabular}{|l|l|}
\hline device & Power usage in watts \\
\hline Normal bulb & 60 \\
\hline CFL bulb & 20 \\
\hline Celling fan & 510 \\
\hline Speaker & 60 \\
\hline Air conditioner & 1200 \\
\hline Computer & 270 \\
\hline TV & 100 \\
\hline Vacuum cleaner & 500 \\
\hline Iron box & 1,100 \\
\hline Washing machine & 700 \\
\hline Cell phone charging & $2-4$ \\
\hline Cameras for security & 25 \\
\hline Electric Clothes Dryer & 3400 \\
\hline Coffee Machine & 1500 \\
\hline Microwave & 1500 \\
\hline Blender & 300 \\
\hline LCD Monitor & $80-150$ \\
\hline Portable Fan & 100 \\
\hline Well Pump (1/3-1 HP) & $480-1200$ \\
\hline Satellite dish & 30 \\
\hline Clothes Dryer - Gas Heated & $300-400$ \\
\hline Hair Dryer & 1,538 \\
\hline Water heater & 479 \\
\hline Shaver & 15 \\
\hline Video Game Player & 195 \\
\hline
\end{tabular}

Figure 2: Power usage by appliances

Some of the electrical appliances shown in above table are widely used in day to day life. The power can be saved by maximizing the use of CFL bulbs, other low power driven devices. The computer and other similar devices will consume only less power in standby mode hence such systems are always neglected when a IoT based power management system is installed .but in a technical university there is a huge number of such electronic devices .so we need to develop specific IoT platforms for university. There are 4 types of IoT platforms, they are M2M Connectivity platforms, and IaaS back ends, Hardware-specific software platforms and Consumer/Enterprise software extensions. 


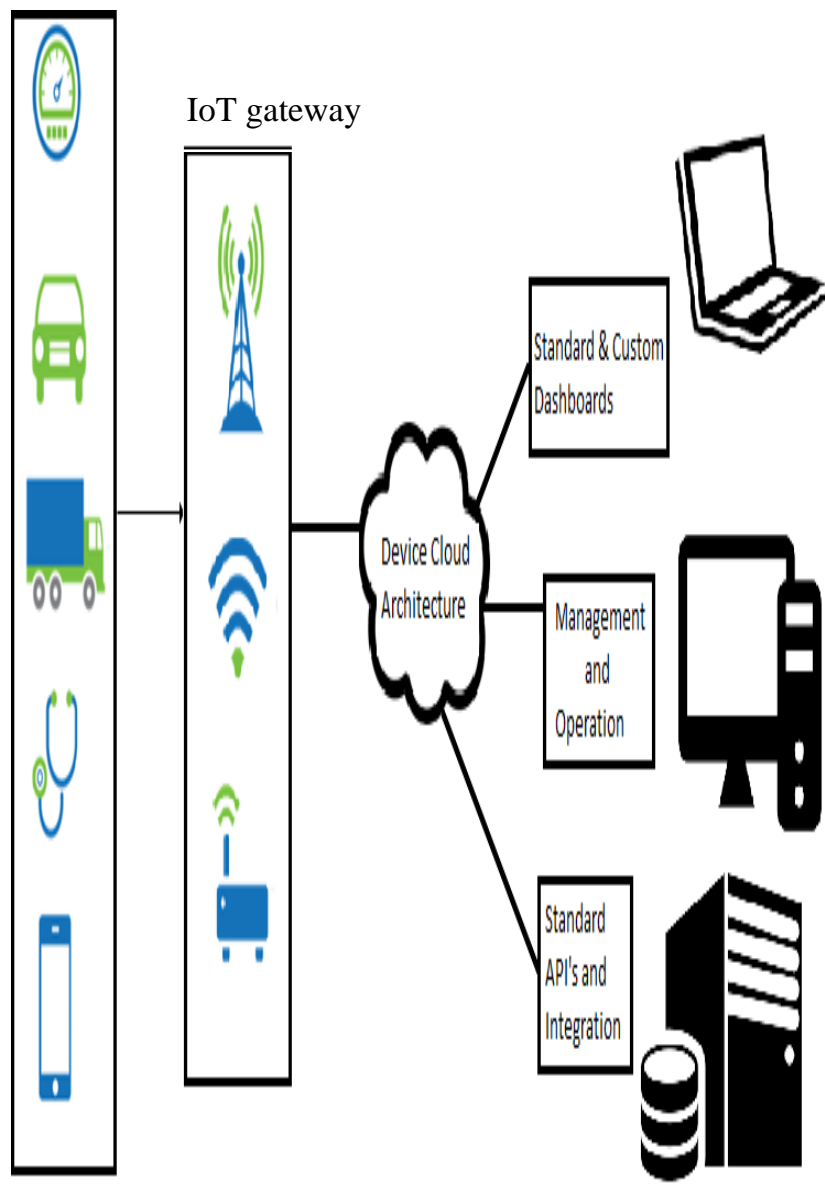

Figure3: A typical M2M BASED IoT architecture.

The above figure shows the elements in the M2M based architecture which is one of the most effective IoT based architecture.

\section{CASE STUDY ON POWER LOSE DURING DISTRIBUTION}

Power losses take place in the progression of distributing electrical energy to users due to technical and commercial losses. The technical losses are due to energy dissipated in the conductors and equipment used for transmission, transformation, sub- transmission and distribution of power. These technical losses are inherent in a system and can be reduced to an optimum level. The losses can be further sub grouped depending upon the stage of power transformation \& transmission system as Transmission Losses $(400 \mathrm{kV} / 220 \mathrm{kV} / 132 \mathrm{kV} / 66 \mathrm{kV})$, as Sub transmission losses $(33 \mathrm{kV} / 11 \mathrm{kV})$ and Distribution losses $(11 \mathrm{kV} / 0.4 \mathrm{kv})$. The commercial losses are caused by pilferage, defective meters, and errors in meter reading and in estimating unmetered supply of energy The power wastage during the transmission is very high the graphs below shows the power lose in rural places of India. The power lose during distribution is one of the very serious concern in power management across the globe.

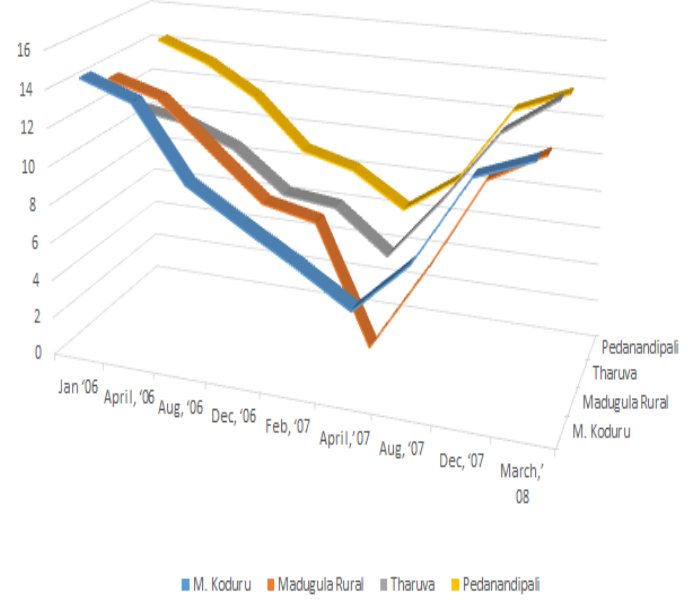

Figure 3: cumulative percentage loss on feeders

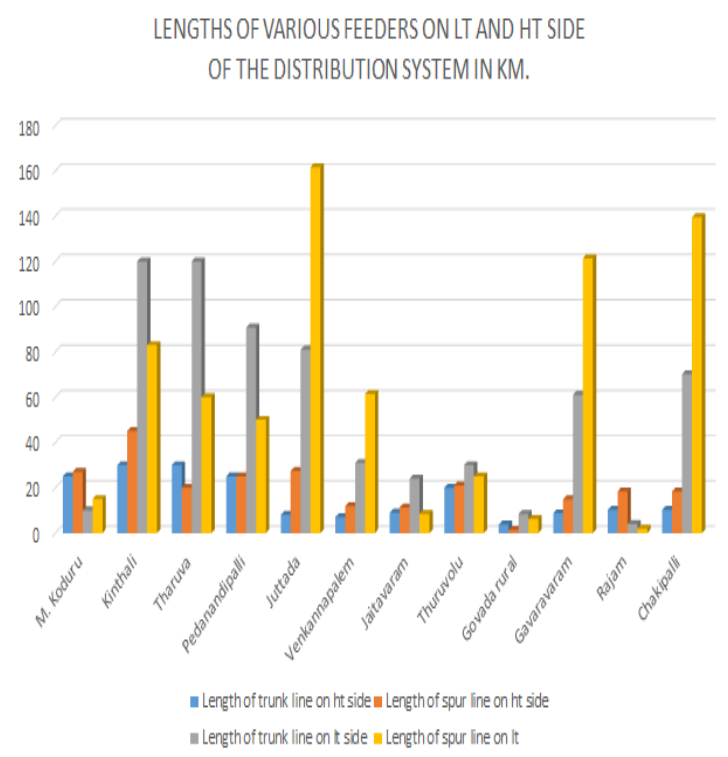

Figure 4: Lengths of various feeders on LT HT side of distribution system in $\mathbf{k m}$

CUMULATIVE PERCENTAGE LOSS ON FEEDERS

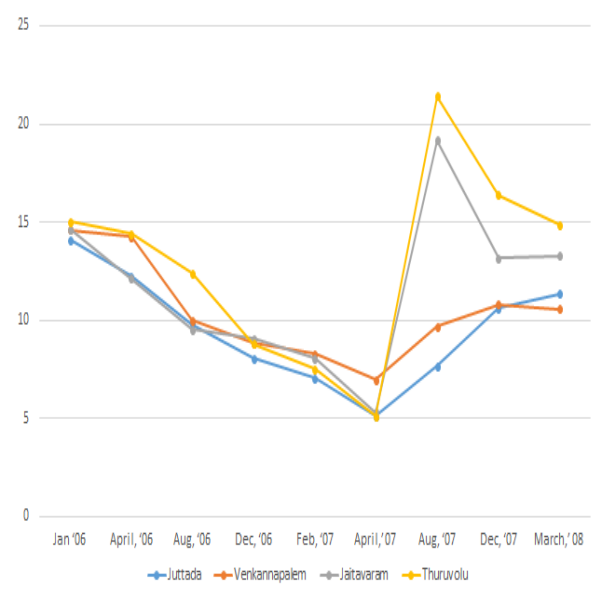

Figure 5: Cumulative percentage loss on feeders 


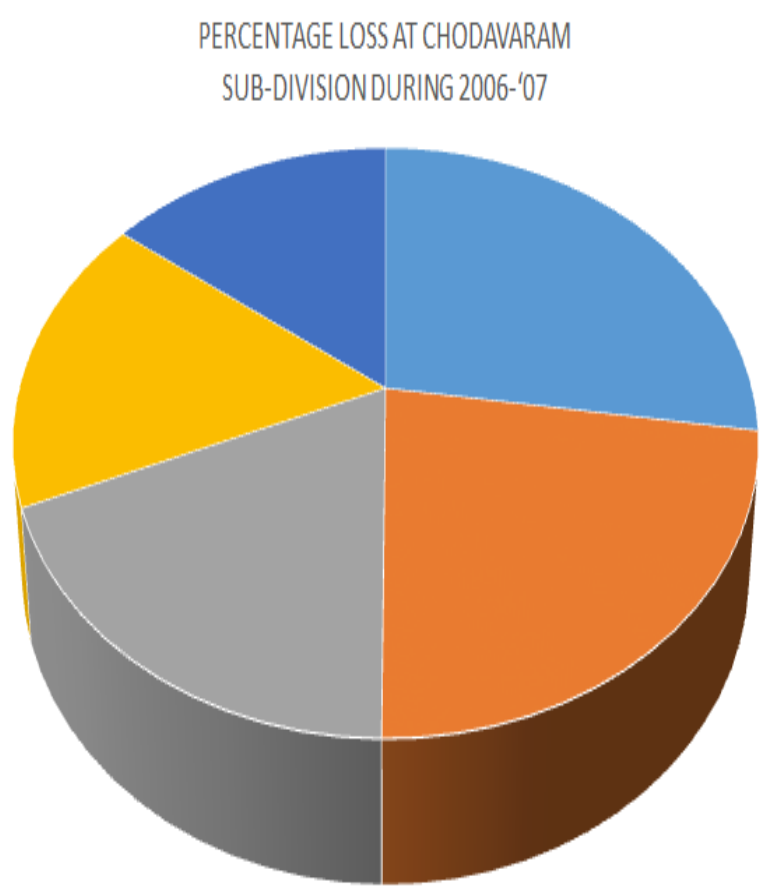

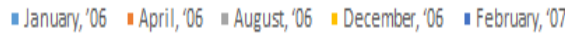

Figure 6: Percentage loss at Chodavaram subdivision during 2006-07

\section{IOT ARCHITECTURE}

There has been present and quite much publicity about "Internet of Things". The notion of a universally interlocked variety of devices, objects and things in universal began with the RFID technology, and this model has significantly been stretched to the present idea that visualizes an excess of mixed things communicating with the physical surroundings. Today, a huge amount of diverse means are used to permit interaction amongst various devices. However, this hard work will lead to a probable strike in inventing a feasible worldwide way out. In addition, current explanations do not talk the flexible necessities for an upcoming IoT; they deliver unsuitable prototypes of supremacy and basically abandonment confidentiality and safe keeping in their project.

There are 4 layers in the IoT. They are Sensor Connectivity and Networks, Gateway and Network, Management Service and Application. Sensor Connectivity and Networks: This includes the Sensor networks, sensors/actuators and Tags. This provides sensor connectivity and network connectivity. Tags which includes RFID, Barcode, and other tag devices. Sensors/Actuators which consists of Attitude indicator, Depth gauge, Gyroscope, GPS, Accelerometer, etc. And LAN which consists of local networking includes Wi-Fi, Ethernet, Bluetooth, ZigBee, Wired, etc. This layer is made up of sensors and smart devices. These sensors are used to collect the real time information and to be processed. Sensor Network put away fewer power and low data rate connectivity so as Wireless Sensor Network (WSN). Sensors are assembled according to their tenacity and data types. Sensor aggregators are the Gateways units. These need to be provided using networking connectivity. At the LAN we have Ethernet and Wi-Fi connections. Ad at the PAN we have ZigBee, Bluetooth and other protocols as well. Sensors which need not be connected to the LAN gateway which can be connected to the Internet by using WAN (Wide Area Network). Gateway and Network: This includes Wide Area network (WAN) or global communication network. That is Wi-Fi, Ethernet and Gateway Control. The Gateway includes Microcontroller, Radio Communication Module, Signal Processor and Modulator, Access point, Embedded /OS, Sim Module, Encryption. The Gateway network connects the gateway and sensor networks together. In this domain we have Wide Area Network (WAN) and Local Area Network (LAN) technologies. This consists of Wi-Fi, Ethernet and Internet.

Gateway and Network layer is the second layer. This must support massive volumes of IoT data produced by wireless sensors and smart devices. It requires robust and reliable performance, regarding private, public or hybrid network models. In addition, Network models are designed to support the communication QoS requirements for latency, error probability, scalability, bandwidth, security, while achieving high levels of energy efficiency meaning that they are low energy efficiency. In addition, it is important to integrate different types of networks into a single IoT platform. IoT sensors are aggregated with various types of protocols and heterogeneous networks using different technologies. IoT network needs to be scalable to efficiently serve a wide range of services and applications overall large scale networks, where in large scale networks some parts may have different packet types and different security requirements. Management Service: This includes Device modeling, Configuration and Management is the main focus. Dataflow management, Security Control is needed to be provided in this layer. Here the management services are Operational Support System (OSS), this includes device modeling, configuration, Management, Performance Management, Security Management. Then there is Billing Support System (BSS) which includes Billing Reporting. Service Analytics Platform is for Statistical Analytics, Data Mining, Text Mining and Management layer for security includes Access control, Encryption, Identify access. In addition, Business Rule Management (BRM) which includes Rule Definition, Modeling, Simulation and Execution. Then there is the Business Process Management (BPM) which has Water flow process, Modeling, Simulation and Execution.

In the Management Service layer, it is in charge of Information Analytics, Security Control, Process Modeling and Device Management. In data management side we need periodic IoT sensor data requires filtering. Some data may not be needed. But because it is periodically going to be collecting information, there is going to be a lot of information which we actually don't need. We throw that unwanted information and we pick only the required information. And use to Actuate, provide control, Management based upon these data. Aperiodic event triggered IoT sensor data may require immediate delivery and response. Application: This includes Energy, Environmental, People Tracking, Retail, Healthcare, Supply Chain, Surveillance, and lot more. In the Horizontal Market it consists of Fleet Management, Asset Management, Supply Chain, People Tracking and Surveillance. The sectors which use this for the overall domain of the application are Sector which includes Environment, Energy, Transport, Healthcare, Retail and Military. And this is the major application domain.

In the Application layer various industry sectors can use IoT for service enhancement. Applications can be classified based on the type of network availability, coverage, size, 
heterogeneity, business model, as well as real-time or nonreal-time requirements. Personal and Home where IoT at the scale of an individual or home. Small limited number of users so overall application needs to focus on some number of users. When we reached the Enterprise here IoT scale of a community is much larger. Then we go to the Utility layer here IoT at a national or regional scale. Then there is a mobile where devices are usually spread across other domains mainly due to their mobility, and lots of the devices we use are battery operated portable IoT devices and therefore they can move around us everywhere with us. That's why mobility support is very important.

IoT has been developing at very swift rate. Owing to this, study on novel IoT devices and IoT wireless equipment is also expanding to carry the IoT goods at low-cost and more rapid rate. IoT has remained categorized into two classifications viz. public to belongings mentioned as $\mathrm{C} 2 \mathrm{~B}$ (Customer to Business) and belongings to belongings or appliance to appliance mentioned as M2M. Public to belongings includes IoT devices obtainable at home such as wearable's, health associated appliances, linked belongings etc. M2M includes everything associated to developed and computerization production. Let us comprehend fundamentals of IoT structural design. As we identify IoT structure contains of three key parts viz. measuring device, network connectivity and data storing applications. The identical has been presented in figure-1. Figure shows, Sensors in the IoT devices one or the other lead into the central server for information storing or transfer via gateway strategies.

Sensors for numerous applications are used in not the same IoT devices according to dissimilar presentations for example temperature, force proximity, power, humidity, etc. Gateway takes attention of numerous wireless standard crossing points and in future one gateway can grip various equipment and several sensors. The classic wireless equipment used broadly is Bluetooth, 6LoWPAN, Zwave, NFC, RFID. ZigBee, etc. Gateway crossing point with cloud using support of wireless or wired equipment such as Wi-Fi, Mobile, DSL or Fiber. As revealed IoT chains in cooperation IPv4 and IPv6 procedures. Owing to support of IPv6 which has nearly 128 bit protracted IP address size, there are sufficient addresses obtainable to rising request of IoT policies. DTN (Delay Tolerant Networks) is the exclusive feature of IoT which receipts care of huge variable interruption necessity of IoT established networks relate to customary computer networks. As displayed, IoT facility suppliers offer different QoS with dissimilar set a price and strategy need for memory, CPU and battery intake.

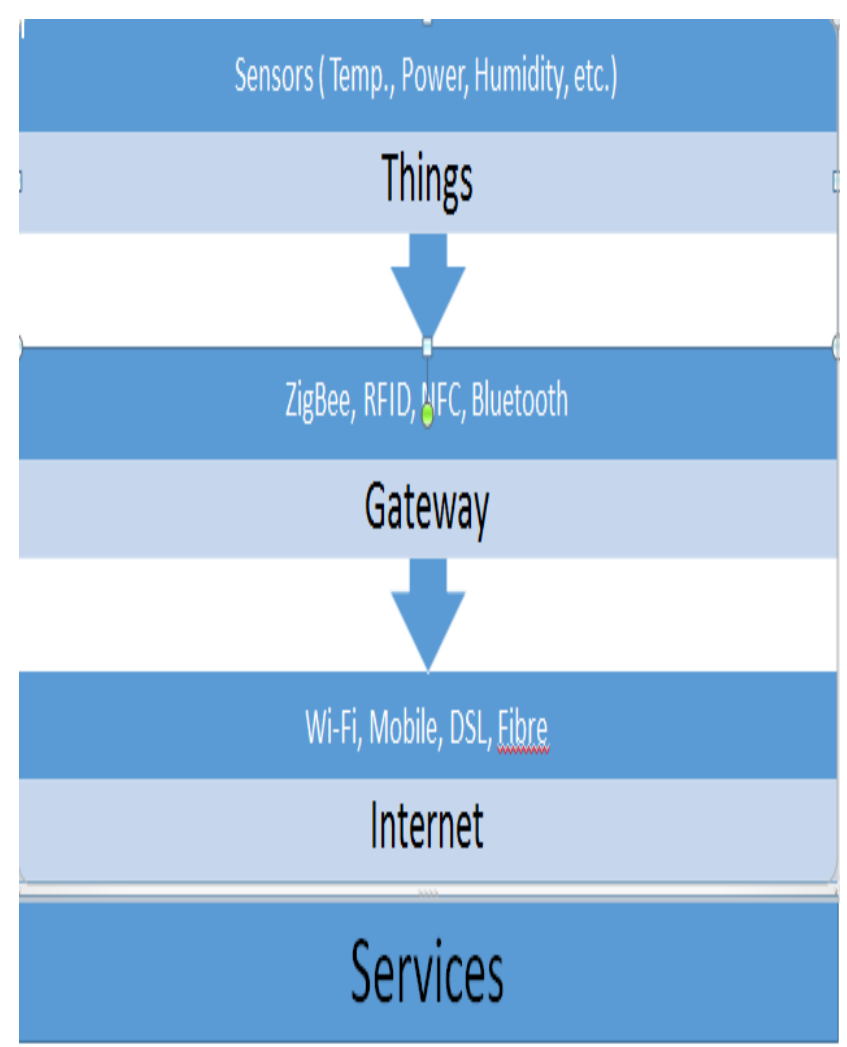

Figure 7: IoT structure

The initial phase of the IoT has previously taken place to alter the way of living, to do commercial services and sort judgments. As prices of regulators, processors, measuring device and transmitter's decline, no matter what that can be assumed an electronic circuit has purpose to distantly lead into other structures are an applicant to develop "smart systems".
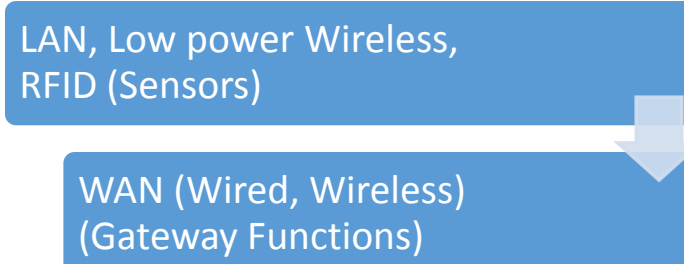

Data, Security, BPM, BRM, Analytics, OSS \& BSS

Applications

Figure 8: IoT technologies 


\section{PROPOSED METHOD}

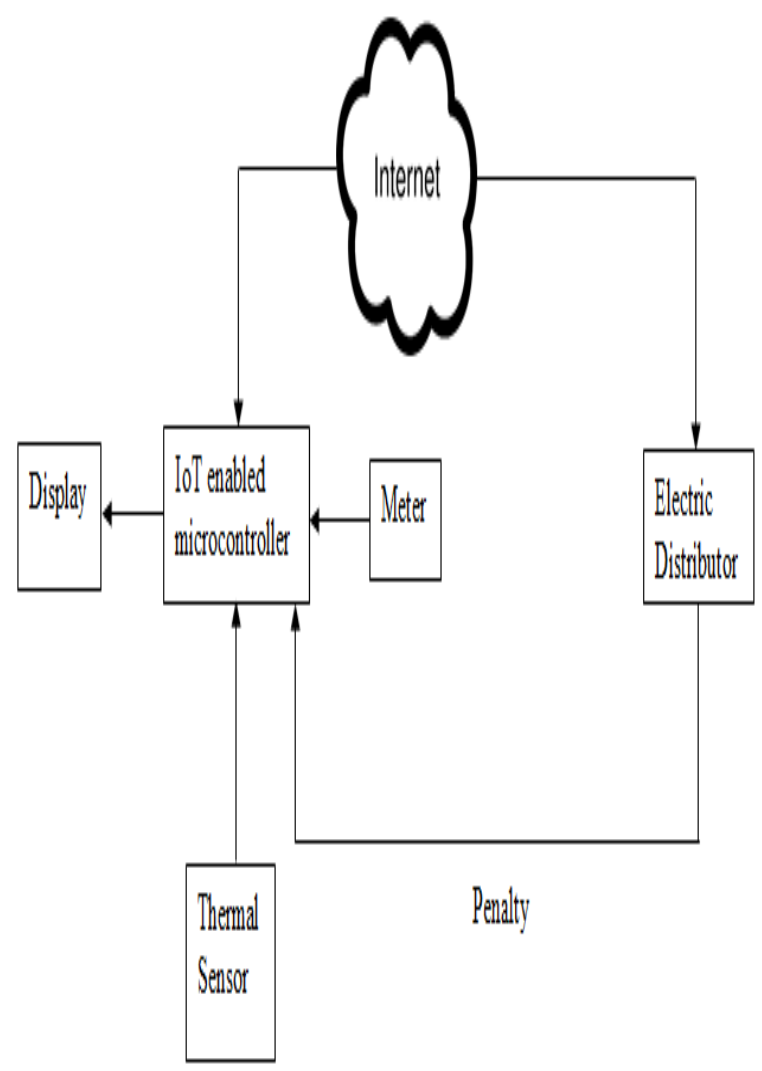

Figure 9: block diagram for proposed method

Display is an output scheme for exhibition of facts in visual or demonstrative form. When the input facts are provided as an electrical indication, the display is called an automatic display. Approximately displays can indication only numerals or alphanumeric typescripts. They are termed segment displays, since they are collected of numerous sections that shift on and off to stretch arrival of wanted glyph. The fragments are typically sole LEDs or liquid crystals. There are numerous types:

- $\quad$ Seven-segment display

- Fourteen-segment display

- $\quad$ Sixteen-segment display

- HD44780 LCD controller

Seven-segment display: Is a method of electrical display device for presenting decimal digits that is an substitute to the additional compound dot matrix displays. These displays are extensively used in alpha numeric watches, electrical meters, simple computers, and additional electronic devices that display mathematical statistics.

Fourteen-segment display is also occasionally mentioned to as a "Union Jack" display. This is a category of display constructed on 14 fragments that can be switched on or off to produce literatures and numbers. It is a development of the extra shared seven-segment display, taking a further four crosswise and two erect sections with the central flat section damaged in half. A seven-segment display suits for digits and definite letters, but unmistakably representation the ISO basic
Latin script needs more aspect. A minor difference is the sixteen-segment display which permits supplementary legibility in exhibiting literatures or additional cyphers.

Sixteen-segment display is a kind of display created on 16 fragments that can be switched on or off permitting to the detailed design to be formed. It is an addition of the extra common seven-segment display, additional four diagonal and two upright fragments and unbearable the three straight fragments in part. Additional alternatives comprise the fourteen-segment display which ensures non division of the top or bottom straight fragments.

Hitachi HD44780 LCD controller is a generally used alphanumeric dot matrix LCD regulator technologically advanced by Hitachi. The governor crossing point and procedure is a de-facto typical for this type of display. The character set of the organizer comprises ASCII typescripts, Japanese Kana fonts, and about cyphers in two 28 character lines. By means of an addition driver, the means can exhibition up to 80 characters.

Thermal Sensor likewise named as an electromagnetic camera or thermal imaging camera is a stratagem that produces a duplicate by means of electromagnetic radiation, like a mutual camera that forms a copy using visible light. In its place of the $400-700 \mathrm{~nm}$ variety of the visible light camera, electromagnetic cameras function in wavelengths as extensive as $14,000 \mathrm{~nm}(14 \mu \mathrm{m})$. Their usage is known as thermography.

Pictures from electromagnetic cameras have a tendency to be neutral since the cameras usually use an image sensor that does not differentiate diverse wavelengths of electromagnetic radiation. Color image measuring device need a composite building to distinguish wavelengths, and color has fewer sense free standing of the standard visible spectrum since the differing wavelengths do not record consistently into the structure of color visualization used by individuals.

Occasionally these monotone images are presented in pseudocolor, where deviations in color are used moderately than deviations in concentration to display variations in the indication. This is valuable since even though individuals have much superior active range in intensity recognition than color general, the capability to see well concentration alterations in perky parts is fairly restricted. This procedure is known as density slicing.

The proposed system uses the thermal sensors which will detect the human presence in the class and other offices of the university by sensing the thermogram. There will be the power measurement device kept in all the rooms. When there are no humans detected, the sensor sends a notification to microcontroller to save the meter reading until human detection is made. If the electronic appliances are working at this time interval, the microcontroller sends a message to the server of power distributors regarding the measurement of power wasted and then power distributors resends the penalty information to the university. 


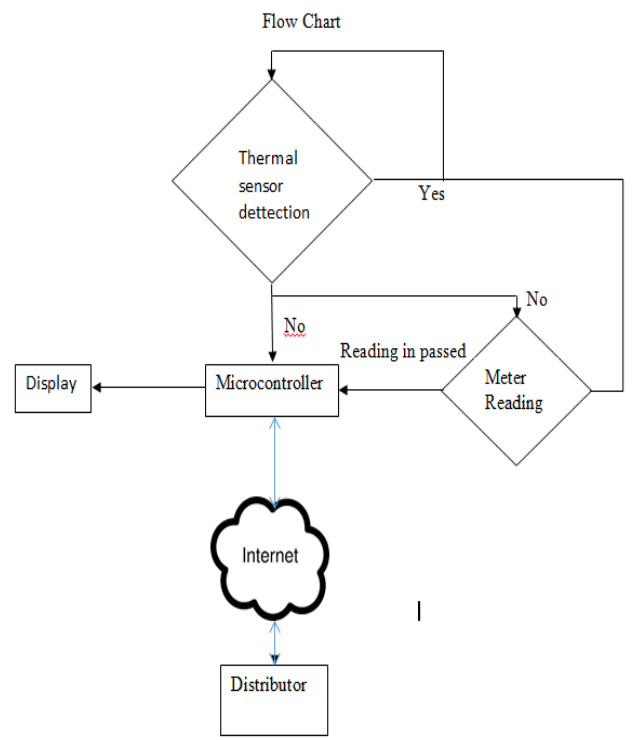

Figure 9: flow chart of proposed method

\section{CONCLUSION}

The present IoT based power management systems using image processing is very costly, hence the paper proposes a cheap method to regulate the wastage of power by giving penalty to the individual or organization by the power distributors the system compromises of thermal sensing and associated hardware, this system requires less installation cost and maintenance is cheap. This method allows the government to control the entire power wastage by remotely and it helps in power saving.

\section{REFERENCES}

[1] K. Elissa, "Title of paper if known," unpublished.

[2] R. Nicole, "Title of paper with only first word capitalized," J. Name Stand. Abbrev., in press.
[3] Y. Yorozu, M. Hirano, K. Oka, and Y. Tagawa, "Electron spectroscopy studies on magneto-optical media and plastic substrate interface," IEEE Transl. J. Magn. Japan, vol. 2, pp. 740-741, August 1987 [Digests 9th Annual Conf. Magnetics Japan, p. 301, 1982].

[4] M. Young, The Technical Writer's Handbook. Mill Valley, CA: University Science, 1989.

[5] Perera c, Zaslavsky A, Christen P, Georgakopoulos D,"Context A ware Computing for The Internet of Things: A Survey", IEEE Communications Surveys \& Tutorials, J6(1),First Quarter 2014.

[6] J. JeyaPadmini,K. R. Kashwan, "Effective Power Utilization and Conservation in Smart Homes Using loT “,2015 international conference on computation of power, energy, information and communication ,2015

[7] T. Guettari y, J. Boudy, BE. Benkelfat, G. Chollet,JL. Baldinger, " Thermal signal analysis in smart home environment for detecting human presence",1st International Conference on Advanced Technologiesfor Signal and Image Processing - ATSIP'2014, March 1719, 2014, Sousse, Tunisia

[8] Ivan Ganchev, Zhanlin Ji, M'airt'in O'Droma," A Generic IoT Architecture for Smart Cities". ISSC 2014 / CIICT 2014, Limerick.

[9] Nihesh Rathod, Pratik Jain and Renu Subramanian,"Performance Analysis of Wireless Devices for a Campus-wide IoT Network". The 2015 International Workshop on Wireless Network Measurements and Experimentation, 2015

[10] Qazi Mamoon Ashraf, Mohamed Hadi Habaebi, Gopinath Rao Sinniah, Musse Mohamud Ahmed, Sheroz Khan," Autonomic Protocol and Architecture for Devices in Internet of Things ", 2014 IEEE innovative smart grid technologies ,Asia(ISGST ,Asia) 